\title{
Degradation Kinetics of Gamma-Oryzanol in Antioxidant-Stripped Rice Bran Oil during Thermal Oxidation
}

\author{
Pramote Khuwijitjaru ${ }^{1 *}$, Thippawan Yuenyong ${ }^{1}$, Rungnaphar Pongsawatmanit ${ }^{2}$ and \\ Shuji Adachi ${ }^{3}$ \\ ${ }^{1}$ Department of Food Technology, Faculty of Engineering and Industrial Technology, Silpakorn University (Nakhon Pathom 73000, \\ THAILAND) \\ ${ }^{2}$ Department of Product Development, Faculty of Agro-Industry, Kasetsart University (Bangkok 10900, THAILAND) \\ ${ }^{3}$ Division of Food Science and Biotechnology, Graduate School of Agriculture, Kyoto University (Kyoto 606-8502, JAPAN)
}

\begin{abstract}
Gamma-oryzanol, a group of phytosterol ferulates found in rice bran, possesses antioxidative activity and other bioactivities. The kinetics of thermal degradation of gamma-oryzanol in stripped rice bran oil (SRBO) were investigated under heating at 132, 160, 192 and $222^{\circ} \mathrm{C}$ for $480,140,60$ and $50 \mathrm{~h}$, respectively. Losses of the overall gamma-oryzanol and its components (cycloartenyl ferulate, 24methylene cycloartanyl ferulate, campesteryl ferulate and $\beta$-sitosteryl ferulate) could be expressed by the first-order kinetics model. The rate constant of thermal degradation of gamma-oryzanol increased with increasing heating temperatures. The temperature dependence of the obtained rate constants was found to obey the Arrhenius equation. Campesteryl ferulate showed slightly more thermally resistant than other components at temperature lower than $160^{\circ} \mathrm{C}$. However, the change in the absorbance from 2,2-diphenyl-1picrylhydrazyl (DPPH) assay as a function of heating time exhibited the same pattern for the SRBO with and without gamma-oryzanol for all studied heating temperatures.
\end{abstract}

Key words: gamma-oryzanol, degradation kinetics, antioxidant-stripped rice bran oil, thermal oxidation, DPPH assay

\section{INTRODUCTION}

Rice bran oil is widely used as cooking oil in Asian countries and gaining acceptance in western countries as well. This oil is well known for its abundance of phytochemicals ${ }^{1)}$ and for its stability as a frying oil ${ }^{2}$. Gamma-oryzanol, a group of ferulic acid esters of phytosterols, is a major phytochemical in rice bran oil. Among the ten components of gamma-oryzanol that have been isolated and identified from rice bran $\mathrm{oil}^{3)}$, the four major components are cycloartenyl ferulate, 24-methylene cycloartanyl ferulate, $\beta$-sitosteryl ferulate and campesteryl ferulate ${ }^{4)}$. The gamma-oryzanol exhibits antioxidant properties, including free radical scavenging ${ }^{4,5}$ and lipoperoxidation prevention ${ }^{5,6)}$. The anticholesterol effect of the compound has also been reported ${ }^{7)}$.

In a cooking process such as deep-fat frying, the oil is heated at high temperature for long period and therefore losses of phytochemicals in the oil can not be avoided.
Thus, information on the stability of the phytochemicals during the cooking process is important for prediction of the remaining amount after the process. The stability of the gamma-oryzanol in rice bran oil during thermal oxidation, however, has not yet been thoroughly studied. We previously showed that the degradation of gamma-oryzanol in unstripped rice bran oil at 120 to $200^{\circ} \mathrm{C}$ could be expressed by a pseudo-first order kinetics model ${ }^{8}$. In our previous report, only overall gamma-oryzanol content was monitored. However, the thermal stability of different components could not be unveiled, although Nyström et $a l^{9}{ }^{9}$ showed that sitostanyl ferulate, a saturated stanol ester, in high oleic sunflower oil was reduced from about $10 \mathrm{mg} / \mathrm{g}$ to about $2 \mathrm{mg} / \mathrm{g}$ after 6 -h oxidation at $180^{\circ} \mathrm{C}$.

The degradation kinetics of gamma-oryzanol and its components in antioxidant-stripped rice bran oil were investigated in this study to gain more understanding the loss of the antioxidants under thermal treatment. 2,2-

\footnotetext{
*Correspondence to: Pramote Khuwijitjaru, Department of Food Technology, Faculty of Engineering and Industrial Technology, Silpakorn University, Nakhon Pathom 73000, THAILAND

E-mail: kpramote@su.ac.th.
}

Accepted May 11, 2009 (received for review April 1, 2009)

Journal of Oleo Science ISSN 1345-8957 print / ISSN 1347-3352 online

http://www.jstage.jst.go.jp/browse/jos/ 
diphenyl-1-picrylhydrazyl (DPPH) assay has been used for determination of antioxidant capacities of various compounds including vegetable oils ${ }^{10)}$. Lee $e t a l .{ }^{11)}$ have used the DPPH assay to predict the oxidative stability of edibles oil. Therefore, the DPPH assay of heated oil was also investigated to determine the relationship between the radical scavenging activity and the presence of gamma-oryzanol in highly thermally oxidized oil.

\section{EXPERIMENTAL}

\subsection{Materials}

Rice bran oil was purchased from a local market in Nakhon Pathom, Thailand. Gamma-oryzanol (purity $>$ 98.5\%) was a gift from Tsuno Rice Fine Chemical Co., Ltd. (Wakayama, Japan). Aluminum oxide (chromatography type, neutral, activity I) was from Fluka (Switzerland) and DPPH was from Sigma-Aldrich (Germany).

\subsection{Preparation of antioxidant-stripped rice bran oil}

To eliminate the effect of natural antioxidants, the rice bran oil was passed through an alumina column to remove them before use in the experiment. This procedure was successful in removal of several pro- and antioxidants in vegetable oils ${ }^{9,12)}$. The method of Nyström et al..$^{9)}$ was followed. A glass column $(480 \times 30 \mathrm{~mm}$ i.d. $)$ was packed with $200 \mathrm{~g}$ of aluminum oxide. A mixture of $100 \mathrm{~g}$ rice bran oil and $100 \mathrm{~mL}$ of hexane was added to the column and eluted with $200 \mathrm{~mL}$ of hexane at $2.5 \mathrm{~mL} / \mathrm{min}$. The effluent was kept at $-18^{\circ} \mathrm{C}$ and the hexane was removed by vacuum evaporation before use. Stripped rice bran oil (SRBO) was analyzed for its fatty acid composition at the National Food Institute laboratory (Bangkok, Thailand) by gas chromatography ${ }^{13)}$.

\subsection{Heating of stripped rice bran oil}

Gamma-oryzanol was added to the SRBO at the concentration of $3,000 \mathrm{mg} / \mathrm{kg}$, which was in the range of that declared in commercial rice bran oil available in Thailand $(3,000-4,000 \mathrm{mg} / \mathrm{kg})$. Samples of the SRBO $(15 \mathrm{~mL})$ were then transferred into several open $25 \mathrm{~mL}$-test tubes (i.d. 2.3 $\mathrm{cm}$; surface area: $4.1 \mathrm{~cm}^{2}$ ) and heated without mixing in a laboratory-made temperature controlled-silicone oil bath at $132,160,192$ and $222^{\circ} \mathrm{C}$. A type- $\mathrm{K}$ thermocouple was connected to the oil bath to measure the temperature. The tested temperatures cover most frying process temperatures. Samples were randomly taken at appropriate interval for chemical analyses until the gamma-oryzanol was degraded more than $90 \%$. Heating of the SRBO at each temperature was performed in duplicate.

\subsection{HPLC determination of gamma-oryzanol}

The amount of gamma-oryzanol remaining in the heated
SRBO was determined by reverse-phase HPLC according to the method of $\mathrm{Xu}$ and Godber ${ }^{3)}$ with some modifications. The instrument was comprised of a 510 high pressure pump, a 486 Tunable Absorbance detector (Waters, Massachusetts, USA) and a C-R5A chart recorder (Shimadzu, Kyoto, Japan). Inertsil ODS-3 column $(250 \times 4.6 \mathrm{~mm}$, GL Sciences, Tokyo, Japan) was used for chromatographic separation. The mobile phase was a mixture of methanol, acetonitrile and acetic acid (55:42:3 by vol.) and its flow rate was $1.8 \mathrm{~mL} / \mathrm{min}$. An oil sample (500 mg) was first diluted with $5.0 \mathrm{~mL}$ of acetone. The solution was filtered through a Nylon syringe filter $(0.45 \mu \mathrm{m})$ and then $20 \mu \mathrm{L}$ of the filtered sample was injected into the HPLC system. Each sample was analyzed in duplicate. The elution profile was monitored at $330 \mathrm{~nm}$. Four major peaks of the gamma-oryzanol components were eluted at the retention times of 22 to 32 min (Fig. 1). Peaks 1 to 4 of gamma-oryzanol components have been identified by the manufacturer to be cycloartenyl ferulate, 24-methylene cycloartanyl ferulate, campesteryl ferulate and $\beta$-sitosteryl ferulate, respectively. The peak area of each component was summed for calculation of the overall gamma-oryzanol concentration. A standard curve of gamma-oryzanol (25 - 400 ppm) was prepared for quantitative analysis.

The effect of the oil matrix in injected sample on the HPLC measurement was evaluated by spiking gammaoryzanol to the SRBO at the concentrations of 3,000 and $300 \mathrm{mg} / \mathrm{kg}$ (approximately lowest concentration in this study), and the gamma-oryzanol concentrations were determined by the procedure mentioned above (five replications). Using a $t$-test method ( $\mathrm{n}=5, \alpha=0.05)$, the deter-

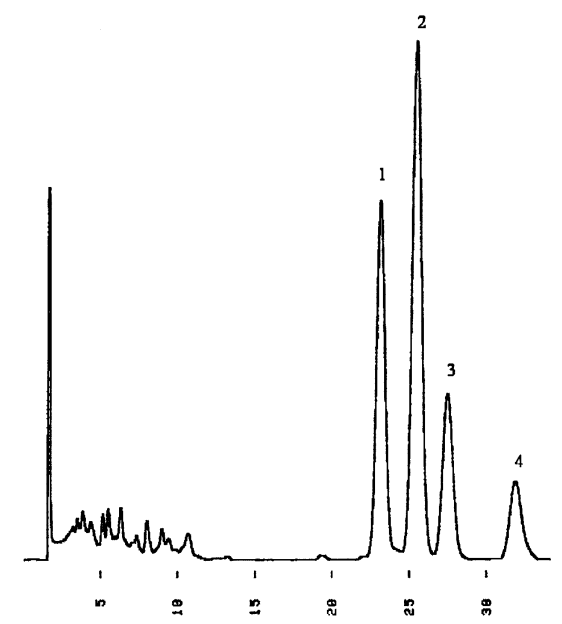

Fig. 1 HPLC Chromatogram of Gamma-Oryzanol (peaks 1 to 4) in Stripped Rice Bran Oil by UV Detector at $330 \mathrm{~nm}$.

Peaks 1 to 4 were identified as cycloartenyl ferulate, 24-methylene cycloartanyl ferulate, campesteryl ferulate and $\beta$-sitosteryl ferulate, respectively. 
mined concentrations were not significantly different from the spiked concentrations $(p>0.18$ and $p>0.55$, respectively). Thus, the effect of oil matrix in the sample could be neglected and direct oil sample injection was used in this study.

\subsection{DPPH assay}

In this study, the DPPH assay of the heated oil was evaluated using ethyl acetate as the solvent ${ }^{14}$. An oil sample $(100 \mu \mathrm{L})$ was mixed with $3.9 \mathrm{~mL}$ of $6 \times 10^{-5} \mathrm{M} \mathrm{DPPH}$, and the absorbance at $515 \mathrm{~nm}$ was measured after 90 min using a Genesys 10 UV spectrophotometer (Thermo Spectronic, USA). Initial absorbance of DPPH solution was about 0.72 . Decreasing of the absorbance would indicate the radical scavenging reaction. Each sample was analyzed in duplicate.

\subsection{Statistical analysis}

The kinetics parameter estimation was done with a nonlinear regression analysis ${ }^{15}$ using the average values from four measurements. Estimated parameters and their standard errors were reported. Statistic analyses of the $t$-test and nonlinear regression were performed using statistical software R version 2.5.16).

\section{RESULTS AND DISCUSSION}

\subsection{Stripping of rice bran oil to remove antioxidants}

Table 1 shows the fatty acid composition of the SRBO. The major fatty acids in the SRBO were oleic (37\%), linoleic (30\%) and palmitic (17\%). The unstripped rice bran used in this study contained gamma-oryzanol at 1,823 \pm 18 $\mathrm{mg} / \mathrm{kg}$ which was lower than the value we expected. Gamma-oryzanol was not detected after stripping using aluminum oxide. The DPPH absorbance for the unstripped rice bran oil was about 0.65 while that for the SRBO was about 0.07 . Thus, the treatment by aluminum oxide

Table 1 Fatty Acid Composition of Stripped Rice Bran Oil.

\begin{tabular}{lc}
\hline \multicolumn{1}{c}{ Fatty acid } & Content $[\%(\mathrm{w} / \mathrm{w})]$ \\
\hline Myristic acid (C14:0) & 0.29 \\
Palmitic acid (C16:0) & 17.20 \\
Stearic acid (C18:0) & 2.08 \\
Arachidic acid (C20:0) & 0.84 \\
Behenic acid (C22:0) & 0.24 \\
Ligoceric acid (C24:0) & 0.38 \\
Palmitoleic acid (C16:1) & 0.24 \\
Oleic acid (C18:1) & 37.39 \\
Linoleic acid (C18:2) & 30.83 \\
Linolenic acid (C18:3) & 0.81 \\
\hline
\end{tabular}

removed most of natural antioxidants presented in the unstripped rice bran oil. Removing of natural antioxidants in vegetable oil using aluminum oxide and its efficiency was reported by several authors ${ }^{9,12,17)}$.

\subsection{Overall degradation of gamma-oryzanol}

Figure 2 shows the degradation processes of the gammaoryzanol at various temperatures. The fraction of the remaining gamma-oryzanol was linearly related to time on a semi-logarithmic scale at every temperature. This fact indicated that the degradation process could be expressed by the first-order kinetics as follows:

$$
C=C_{0} \exp (-k t)
$$

or in linearized form,

$$
\ln C=\ln C_{0}-k t
$$

where $C$ is the concentration of gamma-oryzanol at time $t$, $C_{0}$ is the initial concentration of gamma-oryzanol and $k$ is the rate constant.

The first order kinetics model is often used for modeling the degradation process of several classes of compounds during a thermal treatment. The model has been also used to describe degradation processes of naturally available gamma-oryzanol in unstripped rice bran oil at 120 to $200^{\circ} \mathrm{C}^{8}$ and that of $\alpha$-tocopherol and $\alpha$-tocotrienol in canola oil at $180^{\circ} \mathrm{C}^{12)}$, although more complex models was reported for the degradation of polyphenols in extra-virgin olive oil at 98 to $180^{\circ} \mathrm{C}^{18)}$.

The rate constant $k$ at each temperature was calculated by a nonlinear regression analysis using Eq. (1) and showed

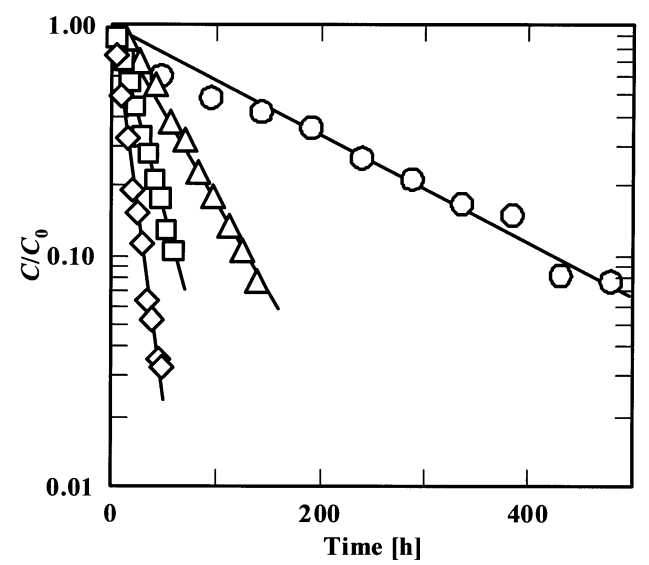

Fig. 2 Degradation of Gamma-Oryzanol in Stripped Rice Bran Oil Heated at $132(\bigcirc), 160(\triangle), 192(\square)$ and $222^{\circ} \mathrm{C}(\diamond)$.

$C$ is the concentration of gamma-oryzanol at any time, and $C_{0}$ is the initial value. 


\section{P. Khuwijitjaru, T. Yuenyong, R. Pongsawatmanit and S. Adachi}

in Table 2. The $k$ value was higher at the higher temperatures, that is, the gamma-oryzanol degraded at faster rate at higher temperature. The temperature dependence of the $k$ value was modeled using the Arrhenius equation:

$$
k=k_{0} \exp \left(-E_{\mathrm{a}} / R T\right)
$$

or in linearized form:

$$
\ln k=\ln k_{0}-E_{\mathrm{a}} / R T
$$

where $T$ is the absolute temperature, $R$ is the gas constant, $k_{0}$ is the pre-exponential factor and $E_{\mathrm{a}}$ is the activation energy. As shown in Fig. 3, the Arrhenius plot using Eq. (4) shows a good linear relationship $\left(R^{2}=0.992\right)$ in the tested temperature range. Calculation of the $E_{\mathrm{a}}$ and $k_{0}$ were performed by substitute Eq. (3) into Eq. (1):

$$
C=C_{0} \exp \left[-k_{0} \exp \left(-E_{\mathrm{a}} / R T\right) \cdot t\right]
$$

and all experimental data (44 values) was used for one-step nonlinear regression. The estimated $E_{\mathrm{a}}$ and $k_{0}$ values were $45.49 \mathrm{~kJ} / \mathrm{mol}$ and $4.70 \times 10^{3} \mathrm{~h}^{-1}$, respectively. The activation energy for degradation of naturally available gammaoryzanol in unstripped rice bran oil was $40.76 \mathrm{~kJ} / \mathrm{mol}^{8)}$ and that of total polyphenols in extra-virgin oil at 98 to $180^{\circ} \mathrm{C}$ was $33-38 \mathrm{~kJ} / \mathrm{mol}^{18}$.

From the kinetics analysis, the results indicated that gamma-oryzanol is quite stable in rice bran oil during thermal oxidation at high temperatures. For example, at $180^{\circ} \mathrm{C}$, which is the ordinary frying temperature, estimated halflife time of the gamma-oryzanol was about $26 \mathrm{~h}$.

Nyström et $a l .^{9)}$ showed that sitostanyl ferulate, a saturated stanol ester, in antioxidant-stripped high oleic sun-

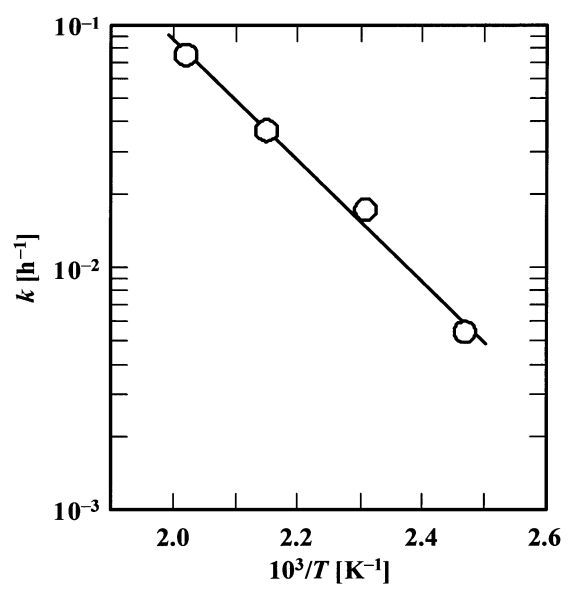

Fig. 3 Temperature Dependence of the Rate Constant $k$ for the Degradation of Gamma-Oryzanol.

$T$ is the absolute temperature. flower oil was reduced from about $10 \mathrm{mg} / \mathrm{g}$ (or 10,000 $\mathrm{mg} / \mathrm{kg}$, i.e. about three times higher than the concentration used in this study) to about $2 \mathrm{mg} / \mathrm{g}$ after $6 \mathrm{~h}$ oxidation at $180^{\circ} \mathrm{C}$. That is, about $80 \%$ of the compound lost in $6 \mathrm{~h}$, which is quite shorter than the value for gamma-oryzanol in this study.

\subsection{Degradation of the components of gamma-oryzanol}

The degradation processes for the components of gamma-oryzanol were also analyzed on the basis of their areas on the HPLC chromatograms (Fig. 4). The degradation of each component at every temperature obeyed the first-order kinetics model:

$$
P=P_{0} \exp (-k t)
$$

or in linearized form,

$$
\ln P=\ln P_{0}-k t
$$

where $P$ is the peak area of gamma-oryzanol component at time $t, P_{0}$ is the initial peak area of gamma-oryzanol component and $k$ is the rate constant. The rate constants, $k$, for each component at different temperatures were evaluated (Table 2). The activation energy and pre-exponential factor for each component were evaluated by the abovementioned method, and are also listed in the table. The activation energy and the pre-exponential factor for campesteryl ferulate were slightly higher than those for other components. This indicated that degradation process of campesteryl ferulate may be different from others. In Fig. 4, at 222 and $192^{\circ} \mathrm{C}$, all the components were degraded at almost the same rate, while at the lower temperatures, campesteryl ferulate showed a slightly lower degradation rate than the other components. Thus, the result suggested that at temperatures lower than $160^{\circ} \mathrm{C}$, campesteryl ferulate was more thermally resistant than the other components. Akiyama et al. . $^{4}$ reported that, however, the DPPH radical scavenging activities of all the four components of gamma oryzanol were almost the same at room temperature. Therefore, a more stable component like campesteryl ferulate may show advantage in protecting the thermal oxidation of lipid for longer period of time.

\subsection{DPPH assay}

Figure 5 shows the changes in the DPPH absorbance for the SRBO during the thermal oxidation at different temperatures. Clearly, the DPPH absorbance did not directly correlate with the amount of gamma-oryzanol in the heated oil. The pattern of change was similar to that reported for lard, soybean, and olive oil with and without $\mathrm{BHT}^{11}$. As expected, the SRBO without gamma-oryzanol resulted in a higher absorbance at $0 \mathrm{~h}$ than the SRBO with gammaoryzanol. The DPPH absorbance for the SRBO both with- 


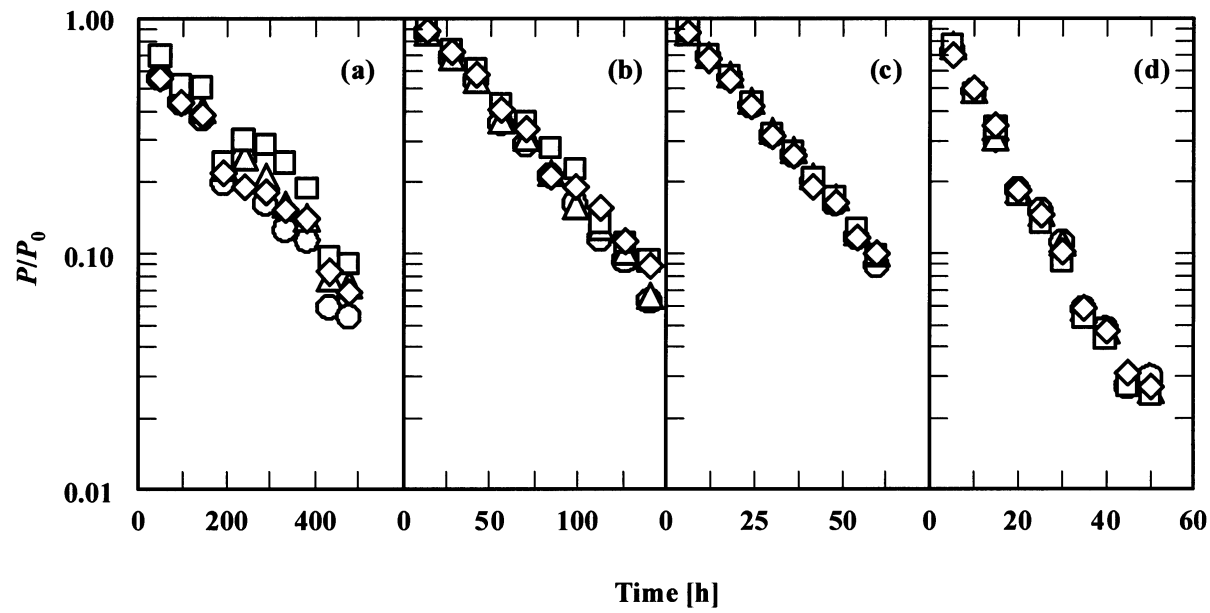

Fig. 4 Changes in Peak Area and Time for Cycloartenyl Ferulate $(\bigcirc)$, 24-Methylene Cycloartanyl Ferulate $(\triangle)$, Campesteryl Ferulate $(\square)$ and $\beta$-Sitosteryl Ferulate $(\diamond)$, which are the Major Components of Gamma-Oryzanol, at (a) $132^{\circ} \mathrm{C}$, (b) $160^{\circ} \mathrm{C}$, (c) $192^{\circ} \mathrm{C}$ and (d) $222^{\circ} \mathrm{C}$.

$P$ and $P_{0}$ are the peak area at any time and the initial value, respectively.

Table 2 Rate Constant $(k)$, Activation Energy $\left(E_{\mathrm{a}}\right)$ and Pre-Exponential Factor $\left(k_{0}\right)$ for the First-Order Degradation Kinetics of the Gamma-Oryzanol and Its Components. Data expressed as estimated value (standard error) from non-linear regression analysis.

\begin{tabular}{cccccc}
\hline & \multicolumn{5}{c}{$k\left[\mathrm{~h}^{-1}\right]$} \\
\cline { 2 - 6 } Temperature $\left[{ }^{\circ} \mathrm{C}\right]$ & Gamma-oryzanol & $\begin{array}{c}\text { Cycloartenyl } \\
\text { ferulate }\end{array}$ & $\begin{array}{c}\text { 24-methylene } \\
\text { cycloartanyl ferulate }\end{array}$ & $\begin{array}{c}\text { Campesteryl } \\
\text { ferulate }\end{array}$ & $\beta$-sitosteryl ferulate \\
\hline 132 & $0.0054(0.0004)$ & $0.0067(0.0006)$ & $0.0059(0.0005)$ & $0.0049(0.0005)$ & $0.0065(0.0006)$ \\
160 & $0.0171(0.0007)$ & $0.0181(0.0008)$ & $0.0176(0.0008)$ & $0.0156(0.0008)$ & $0.0166(0.0008)$ \\
192 & $0.0365(0.0012)$ & $0.0378(0.0011)$ & $0.0364(0.0011)$ & $0.0371(0.0013)$ & $0.0380(0.0011)$ \\
222 & $0.0753(0.0023)$ & $0.0774(0.0021)$ & $0.0767(0.0030)$ & $0.0778(0.0031)$ & $0.0758(0.0019)$ \\
\hline$E_{\mathrm{a}}\left[\mathrm{kJ} / \mathrm{mol}^{3}\right]$ & $45.49(1.20)$ & $42.49(1.34)$ & $44.26(1.22)$ & $47.89(1.34)$ & $44.21(1.13)$ \\
$k_{0}\left[\times 10^{3} \mathrm{~h}^{-1}\right]$ & $4.70(1.53)$ & $2.27(0.82)$ & $3.48(1.15)$ & $8.66(3.15)$ & $3.48(1.07)$ \\
\hline
\end{tabular}

out and with gamma-oryzanol increased during the early stage of the oxidation and then decreased with time. This pattern suggested that gamma-oryzanol (and/or other remaining antioxidants) reacted first with the lipid oxidation free radicals, and then the remaining amount began to react with the DPPH radical. When the oxidation process further progressed, gamma-oryzanol (and/or other remaining antioxidants), however, seemed unable to react with all the lipid oxidation free radicals. Therefore, the free radicals could directly reacted with the DPPH which then resulted in the decrease of DPPH absorbance ${ }^{11}$. Because the higher temperature accelerate the degradation of gamma-oryzanol as discussed in the previous sections and also generally increase the rate of lipid oxidation, the pattern of DPPH absorbance began to change sooner, that is,
$10,12,28$ and $48 \mathrm{~h}$ at $222,192,160$ and $132^{\circ} \mathrm{C}$, respectively. These results also revealed that the DPPH assay could not be immediately used to evaluate the radical scavenging capacity of highly oxidized oil.

\section{CONCLUSION}

Degradation of four major components of gammaoryzanol in antioxidant-stripped rice bran oil could be expressed by the first-order kinetics model at any tested temperature from 132 to $222^{\circ} \mathrm{C}$. The rate constant of thermal degradation of gamma-oryzanol increased with increasing heating temperatures. Activation energy and frequency factor for the degradation of gamma-oryzanol 


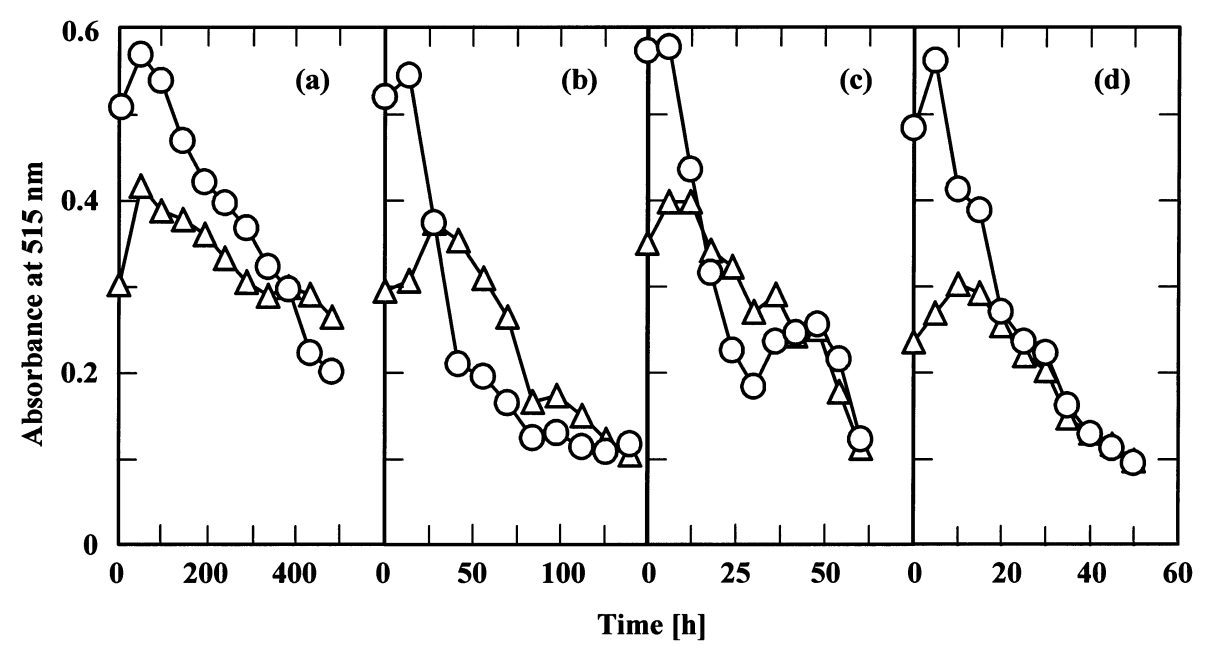

Fig. 5 Change of DPPH Absorbance at $515 \mathrm{~nm}$ after Adding Stripped Rice Bran Oil Heated for Different Periods of Time without $(\bigcirc)$ and with $(\triangle)$ Gamma-Oryzanol at (a) $132^{\circ} \mathrm{C}$, (b) $160^{\circ} \mathrm{C}$, (c) $192^{\circ} \mathrm{C}$ and (d) $222^{\circ} \mathrm{C}$.

and its components could be evaluated based on the Arrhenius equation. The stability of campesteryl ferulate was slightly higher than other components at temperature lower than $160^{\circ} \mathrm{C}$.

\section{ACKNOWLEDGEMENT}

This work was supported by grants from the Thailand Research Fund (MRG5080022) and the Silpakorn University Research and Development Institute.

\section{References}

1. Cheruvanky, R. Bioactives in rice bran and rice bran oil. in Phytochemicals as Bioactive Agents (Bidlack, W.R.; Omaye, S.T.; Meskin, M.S.; Topham, D.K.W., ed). CRC Press, Boca Raton, Florida, pp. 213-240 (2000).

2. Kochhar, S.P. Stabilisation of frying oils with natural antioxidative components. Eur. J. Lipid Sci. Technol. 102, 552-559 (2000).

3. Xu, Z.; Godber, J.S. Purification and identification of components of $\gamma$-oryzanol in rice bran oil. J. Agric. Food Chem. 47, 2724-2728 (1999).

4. Akiyama, Y.; Hori, K.; Takahashi, T.; Yoshiki, Y. Free radical scavenging activities of $\gamma$-oryzanol constituents. Food Sci. Technol. Res. 11, 295-297 (2005).

5. Juliano, C.; Cossu, M.; Alamanni, M.C.; Piu, L. Antioxidant activity of gamma-oryzanol: Mechanism of action and its effect on oxidative stability of pharmaceutical oils. Int. J. Pharm. 299, 146-154 (2005).

6. Xu, Z.; Godber, J. Antioxidant activities of major com- ponents of $\gamma$-oryzanol from rice bran using a linoleic acid model. J. Am. Oil Chem. Soc. 78, 645-649 (2001).

7. Wilson, T.A.; Nicolosi, R.J.; Woolfrey, B.; Kritchevsky, D. Rice bran oil and oryzanol reduce plasma lipid and lipoprotein cholesterol concentrations and aortic cholesterol ester accumulation to a greater extent than ferulic acid in hypercholesterolemic hamsters. J. Nutr. Biochem. 18, 105-112 (2007).

8. Khuwijitjaru, P.; Taengtieng, N.; Changprasit, S. Degradation of gamma-oryzanol in rice bran oil during heating: An analysis using derivative UV-spectrophotometry. Silpakorn Univ. Int. J. 4, 154-165 (2004).

9. Nyström, L.; Achrenius, T.; Lampi, A.-M.; Moreau, R.A.; Piironen, V. A comparison of the antioxidant properties of steryl ferulates with tocopherol at high temperatures. Food Chem. 101, 947-954 (2007).

10. Espin, J.C.; Soler-Rivas, C.; Wichers, H.J. Characterization of the total free radical scavenger capacity of vegetable oils and oil fractions using 2,2-diphenyl-1-picrylhydrazyl radical. J. Agric. Food Chem. 48, 648-656 (2000).

11. Lee, J.; Chung, H.; Chang, P.-S.; Lee, J. Development of a method predicting the oxidative stability of edible oils using 2,2-diphenyl-1-picrylhydrazyl (DPPH). Food Chem. 103, 662-669 (2007).

12. Romero, N.; Robert, P.; Masson, L.; Ortiz, J.; Gonzalez, K.; Tapia, K.; Dobaganes, C. Effect of $\alpha$-tocopherol, $\alpha$ tocotrienol and Rosa mosqueta shell extract on the performance of antioxidant-stripped canola oil (Brassica sp.) at high temperature. Food Chem. 104, 383-389 (2007).

13. AOAC. Official methods of analysis of AOAC International 18th ed. AOAC International. Arlington, VA. 
(2005).

14. Rossi, M.; Alamprese, C.; Ratti, S. Tocopherols and tocotrienols as free radical-scavengers in refined vegetable oils and their stability during deep-fat frying. Food Chem. 102, 812-817 (2007).

15. Van Boekel, M.A.J.S. Statistical aspects of kinetic modeling for food science problems. J. Food Sci. 61, 477486 (1996).

16. $\mathrm{R}$ Development Core Team. $R$ : A language and environment for statistical computing. R Foundation for
Statistical Computing. Vienna, Austria. ISBN 3900051-07-0, URL http://www.R-project.org. (2007).

17. Lampi, A.M.; Dimberg, L.H.; Kamal-Eldin, A. A study on the influence of fucosterol on thermal polymerisation of purified high oleic sunflower triacylglycerols. $J$. Sci. Food Agric. 79, 573-579 (1999).

18. Campanella, L.; Nuccilli, A.; Tomassetti, M.; Vecchio, S. Biosensor analysis for the kinetic study of polyphenols deterioration during the forced thermal oxidation of extra-virgin olive oil. Talanta 74, 1287-1298 (2008). 